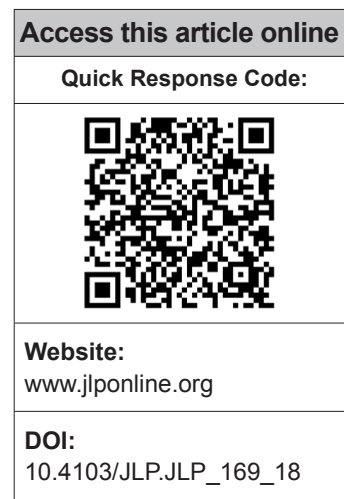

Department of Allied Health Sciences, College of Medicine and Health Sciences, Sultan Qaboos University, Muscat, Oman

Address for correspondence: Dr. Nasar Yousuf Alwahaibi,

Department of Allied Health Sciences, College of Medicine and Health Sciences, Sultan Qaboos University, P.O. Box 35,

Postal Code 123,

Muscat, Oman

E-mail: nasar@squ.edu.

om

Submission: 11-12-2018 Accepted: 22-03-2019

\title{
A substitute to xylene in deparaffinization and clearing prior to coverslipping in histopathology
}

\author{
Nasar Yousuf Alwahaibi, Sirin Hamed Aldughaishi
}

\section{Abstract:}

INTRODUCTION: Deparaffinization and clearing prior to coverslipping are important steps in all staining methods in histopathology. Xylene is the most commonly used agent worldwide. However, xylene is toxic. We evaluated safer alternative dewaxing and clearing agents prior to coverslipping in a histopathology laboratory.

MATERIALS AND METHODS: Thirteen different fresh surgical tissues were cut into two halves. One half processed using xylene and the other half processed using UltraClear ${ }^{\mathrm{TM}}$. Five groups were designed. For each Group of A, B, C, and D, 100 slides were cut from xylene-processed blocks. For Group E, 100 slides were cut from UltraClear ${ }^{\mathrm{TM}}$-processed blocks. Group A is the standard method. Group B evaluates UltraClear ${ }^{\mathrm{TM}}$ as a dewaxing agent only. Group $\mathrm{C}$ evaluates UltraClear ${ }^{\mathrm{TM}}$ as a clearing agent prior to coverslipping only. Group D evaluates UltraClear ${ }^{\mathrm{TM}}$ as both dewaxing and clearing agents prior to coverslipping. Group E evaluates UltraClear ${ }^{\mathrm{TM}}$ as both dewaxing and clearing agents prior to coverslipping. Six parameters were evaluated: nuclear staining, cytoplasmic staining, cell morphology, clarity of staining, uniformity of staining, and cost.

RESULTS: Groups B, C, and D showed 79\% ( $P=0.054), 83 \%(P=0.221)$, and $80 \%(P=0.079)$ adequacy when compared with Group A (89\%), respectively. However, Group E showed only $76 \%(P=0.016)$ adequacy. UltraClear ${ }^{\mathrm{TM}}$ is more expensive than xylene.

CONCLUSION: UltraClear ${ }^{\mathrm{TM}}$ is a promising dewaxing agent. It is also a good clearing agent for use prior to coverslipping in histopathology laboratory. Cost-benefit balance between safety of laboratory workers, good quality staining, and cost-effective strategy needs to be further studied.

Key words:

Clearing agent prior to coverslipping, dewaxing agent, histopathology, UltraClear ${ }^{\mathrm{TM}}$, xylene

\section{Introduction}

T Tistopathology laboratory contains a lot 1 Lof chemicals; most of them are toxic and harmful to histologists and, to a lesser extent, histopathologists. Xylene is one of these chemicals. It has three main functions in a histopathology laboratory. The first use of xylene is as a clearing agent in histoprocessing steps. The goal of clearing is to remove dehydrating agents from tissues and prepare those tissues for embedding agent. The second use is as a dewaxing agent

This is an open access journal, and articles are distributed under the terms of the Creative Commons Attribution-NonCommercial-ShareAlike 4.0 License, which allows others to remix, tweak, and build upon the work non-commercially, as long as appropriate credit is given and the new creations are licensed under the identical terms.

For reprints contact: reprints@medknow.com to remove paraffin wax from the sectioned slides. The third use is to remove alcohols from stained slides before coverslipping the slides, as we call it throughout this study "clearing prior to coverslipping." Other less uses of xylene include cleaning microscopic slides and recycling of used slides. ${ }^{[1]}$

Despite the continuous use of xylene for $>150$ years, it is toxic and hazardous to health. Long-term exposure of xylene affects central nervous system and may lead to memory loss, unconsciousness, and coma. ${ }^{[2]}$ Other reported toxic effects of xylene include cardiac and kidney injuries, fatal blood dyscrasias, skin erythema, and secondary

How to cite this article: Alwahaibi NY, Aldughaishi SH. A substitute to xylene in deparaffinization and clearing prior to coverslipping in histopathology. J Lab Physicians 2019;11:118-22. 
infection $\cdot^{[3,4]}$ In addition, xylene is highly flammable and volatile, which has a low boiling point $\left(137^{\circ} \mathrm{C}-143^{\circ} \mathrm{C}\right)$, a flash point $\left(25^{\circ} \mathrm{C}\right)$, and an ignition point $\left(25^{\circ} \mathrm{C}\right){ }^{[2]}$

UltraClear ${ }^{\mathrm{TM}}$ is a colorless, odorless isoparaffin-based liquid that contains $\mathrm{C} 11-\mathrm{C} 12$ hydrocarbons that are derived from crude oil fractionation and cracking operations. It is environment-friendly, and so far, no toxic effects have been found associated with its use on humans. ${ }^{[5]}$ In addition, UltraClear ${ }^{\mathrm{TM}}$ has a flash point $\left(56^{\circ} \mathrm{C}\right)$ and a boiling point $\left(173^{\circ} \mathrm{C}-193^{\circ} \mathrm{C}\right) \cdot{ }^{[5]} \mathrm{We}$ have recently demonstrated that UltraClear ${ }^{\mathrm{TM}}$ could be used as a routine clearing agent in histopathology laboratory. ${ }^{[6]}$ In this study, we aimed to evaluate the other uses of UltraClear ${ }^{\mathrm{TM}}$ as dewaxing and clearing agents prior to coverslipping in a histopathology laboratory.

\section{Materials and Methods}

Thirteen different fresh surgical tissues were obtained from the Department of Pathology at Sultan Qaboos University Hospital, Oman. We tested samples of the placenta, ovary, prostate, fallopian tube, sebaceous cyst, testis, lipoma, uterus, lung, kidney, omentum, small intestine, and fibroid. Each specimen was cut in half to create two groups. One group was processed using xylene (Fisher Chemical, Leicestershire, UK), and the other group was processed using UltraClear ${ }^{\mathrm{TM}}$ (Avantor's J. T. Baker, Deventer, The Netherlands). All tissues were fixed in 10\% neutral-buffered formalin for $24 \mathrm{~h}$. All tissues were processed as previously described using an automated histoprocessor (Spin Tissue Processor Microm STP 120; Thermo Scientific, Walldorf, Germany). ${ }^{[6]}$ One hundred blocks were prepared, and four slides from each xylene-processed blocks and one slide from each UltraClear ${ }^{\mathrm{TM}}$-processed block were cut at $3 \mu \mathrm{m}$ using a rotatory microtome (Leica RM2135, Nussloch, Germany).

To evaluate the efficiency of UltraClear ${ }^{\mathrm{TM}}$, as dewaxing and clearing agents prior to coverslipping, five groups were designed. We used 100 slides for each group [Table 1]. As recommended by the manufacture, UltraKitt $^{\mathrm{TM}}$ mounting medium (3921, J. T. Baker, Avantor Performance Materilas, Poland) was used for Groups C, D, and E. Distyrene plasticizer xylene $(360294 \mathrm{H}, \mathrm{BDH}$, VWR International Ltd, UK) mounting medium was used for Groups A and B.

All slides were stained with hematoxylin and eosin ( $\mathrm{H}$ and E) [Table 2]. ${ }^{[7]}$ All slides were examined by light microscopy (Olympus, BX 51, Tokyo, Japan). Nuclear and cytoplasmic staining, cell morphology, clarity of staining, and uniformity of staining criteria were used to assess the quality of all sections [Table 3]. ${ }^{[8]}$ Evaluation was performed blindly by three senior biomedical scientists who work in our histopathology laboratory.
Table 1: Study design

\begin{tabular}{|c|c|}
\hline Groups & Description \\
\hline \multirow[t]{3}{*}{$\bar{A}$} & Xylene processed \\
\hline & Xylene dewaxed \\
\hline & Xylene cleared \\
\hline \multirow[t]{3}{*}{ B } & Xylene processed \\
\hline & UltraClear ${ }^{\mathrm{TM}}$ dewaxed \\
\hline & Xylene cleared \\
\hline \multirow[t]{3}{*}{ C } & Xylene processed \\
\hline & Xylene dewaxed \\
\hline & UltraClear ${ }^{\mathrm{TM}}$ cleared \\
\hline \multirow[t]{3}{*}{ D } & Xylene processed \\
\hline & UltraClear'TM dewaxed \\
\hline & UltraClear ${ }^{\mathrm{TM}}$ cleared \\
\hline \multirow[t]{3}{*}{$E$} & UltraClear'TM processed \\
\hline & UltraClear'TM dewaxed \\
\hline & UltraClear ${ }^{\mathrm{TM}}$ cleared \\
\hline
\end{tabular}

The cost of UltraClear ${ }^{\mathrm{TM}}$ and xylene was obtained from local suppliers.

A score of 0 and 1 was given to each parameter and then scores were added up to be graded. If the score was $\leq 2$, graded as inadequate and if the score was 3-5, graded as adequate. ${ }^{[9]}$ The data were analyzed using the Statistical Package for the Social Sciences (SPSS) software version 23 (SPSS Inc., Chicago, IL, USA). Paired Chi-Square test was used to compare the two measurements of the variable. $P<0.05$ was considered statistically significant. The study was approved by the Medical Research Ethics Committee from the College of Medicine and Health Sciences, Sultan Qaboos University, Oman (MREC \# 1118).

\section{Results}

The results of all groups are shown in Table 4 . When the scores were summed and evaluated, there is no statistical difference in the adequacy for diagnosis between Group A compared to Groups B, C, and D. This indicates that UltraClear ${ }^{\mathrm{TM}}$ is equivalent to xylene in dewaxing and clearing prior to coverslipping. However, a significant difference between Group E to the standard was found, which indicates some difference in the efficiency of UltraClear ${ }^{\mathrm{TM}}$ compared to xylene when used as both dewaxing and clearing prior to coverslipping [Table 5]. An example of nuclear staining for all groups in kidney tissues is shown in Figure 1. UltraClear ${ }^{\mathrm{TM}}$ is more expensive than xylene. One liter of xylene costs two Omani rial (OMR) which is equivalent to 5.19 USD whereas UltraClear ${ }^{\mathrm{TM}}$ costs 4.1 OMR equivalent to 10.64 USD.

\section{Discussion}

Histologists are usually exposed to xylene during histoprocessing, deparaffinization, and clearing prior to 
Table 2: Hematoxylin and eosin staining method for all groups

\begin{tabular}{|c|c|c|c|c|c|}
\hline Main steps & Group A & Group B & Group C & Group D & Group E \\
\hline Deparaffinization & Xylene (2) & UltraClearTM (2) & Xylene (2) & UltraClear'TM (2) & UltraClearTM (2) \\
\hline Rehydration & $\begin{array}{l}100 \%, 100 \%, 95 \% \text {, } \\
70 \% \text { alcohol }\end{array}$ & $\begin{array}{l}100 \%, 100 \%, 95 \% \\
70 \% \text { alcohol }\end{array}$ & $\begin{array}{l}100 \%, 100 \%, 95 \% \\
70 \% \text { alcohol }\end{array}$ & $\begin{array}{l}100 \%, 100 \%, 95 \% \\
70 \% \text { alcohol }\end{array}$ & $\begin{array}{l}100 \%, 100 \%, 95 \% \\
70 \% \text { alcohol }\end{array}$ \\
\hline Nuclear staining & Harris hematoxylin & Harris hematoxylin & Harris hematoxylin & Harris hematoxylin & Harris hematoxylin \\
\hline Differentiation & $1 \%$ acid alcohol & $1 \%$ acid alcohol & $1 \%$ acid alcohol & $1 \%$ acid alcohol & $1 \%$ acid alcohol \\
\hline Bluing & $0.2 \%$ ammonia water & $0.2 \%$ ammonia water & $0.2 \%$ ammonia water & $0.2 \%$ ammonia water & $0.2 \%$ ammonia water \\
\hline Cytoplasmic staining & $1 \%$ eosin & $1 \%$ eosin & $1 \%$ eosin & $1 \%$ eosin & $1 \%$ eosin \\
\hline Dehydration & $\begin{array}{l}70 \%, 95 \%, 100 \% \\
100 \% \text { alcohol }\end{array}$ & $\begin{array}{l}70 \%, 95 \%, 100 \% \\
100 \% \text { alcohol }\end{array}$ & $\begin{array}{l}70 \%, 95 \%, 100 \% \\
100 \% \text { alcohol }\end{array}$ & $\begin{array}{l}70 \%, 95 \%, 100 \% \\
100 \% \text { alcohol }\end{array}$ & $\begin{array}{l}70 \%, 95 \%, 100 \% \\
100 \% \text { alcohol }\end{array}$ \\
\hline Clearing prior to coverslipping & Xylene & Xylene & UltraClear ${ }^{\mathrm{TM}}$ & UltraClear ${ }^{\mathrm{TM}}$ & UltraClear'TM \\
\hline Coverslipping & $\mathrm{DPX}$ & DPX & UltraKitt & UltraKitt & UltraKitt \\
\hline $\begin{array}{l}\text { Approximate overall time } \\
\text { required in minutes }\end{array}$ & 42 & 46 & 46 & 46 & 46 \\
\hline
\end{tabular}

Table 3: Evaluation criteria and scoring ${ }^{[8]}$

\begin{tabular}{|c|c|c|}
\hline \multirow{3}{*}{$\begin{array}{l}\text { Features } \\
\text { Nuclear staining }\end{array}$} & \multicolumn{2}{|c|}{ Score and criteria } \\
\hline & Acceptable $=1$ & Unacceptable $=0$ \\
\hline & Round, smooth, and clear nuclear membrane & Granular, disintegrated, and out of focus \\
\hline \multirow[t]{2}{*}{ Cytoplasmic staining } & Acceptable $=1$ & Unacceptable $=0$ \\
\hline & Intact cytoplasmic membrane and transparent cytoplasm & $\begin{array}{l}\text { Disintegrated cytoplasmic membrane, granular cytoplasm } \\
\text { and out of focus }\end{array}$ \\
\hline \multirow[t]{2}{*}{ Cell morphology } & Preserved $=1$ & Unpreserved $=0$ \\
\hline & Absence of folds, no overlap and maintained $\mathrm{N}$ : $\mathrm{C}$ ratio & Overlapping cells, folded and disintegrated cells \\
\hline \multirow{2}{*}{ Clarity of staining } & Present $=1$ & Absent $=0$ \\
\hline & Crispness in staining and transparency & Obliterates the nucleus and cytoplasm \\
\hline \multirow[t]{2}{*}{ Uniformity of staining } & Present $=1$ & Absent $=0$ \\
\hline & Uniformly stained throughout the individual cell & Stained in different shades of color in an individual cell \\
\hline
\end{tabular}

$\mathrm{N}$ : C ratio=Nucleus-cytoplasm ratio

coverslipping. ${ }^{[10]}$ Worldwide, xylene is considered to be the most commonly used dewaxing and clearing agent as it renders tissue transparent and removes alcohols from tissues rapidly. ${ }^{[9]}$ Other less used solutions include chloroform, benzene, toluene, varies types of oils, and commercially available solutions such as Histo-Clear ${ }^{\circledR}$, Sub- $\mathrm{X}^{\circledR}$, Bioclear ${ }^{\circledR}$, Shandon Xylene Substitute ${ }^{\circledR}$, and UltraClear $^{\mathrm{TM}}$. To the best of our knowledge, UltraClear ${ }^{\mathrm{TM}}$ has not yet been tested for use as dewaxing and clearing agents prior to coverslipping in a histopathology laboratory.

The findings of this study show that UltraClear ${ }^{\mathrm{TM}}$ as a dewaxing agent (Group B) was efficient in nuclear staining, cell morphology, and uniformity of staining in comparison with the standard method. This might indicate that UltraClear ${ }^{\mathrm{TM}}$ is effective in removing wax from the tissue sections. However, cytoplasm staining showed a slight significant difference $(P=0.048)$. When all evaluation criteria were summed, UltraClear ${ }^{\mathrm{TM}}$ as a dewaxing agent was adequate.

Very few studies have tested a safer alternative clearing prior to coverslipping agent to xylene. In the current study, adequate cytoplasm staining, preserved cell morphology, and consistent uniformity of staining were seen with UltraClear ${ }^{\mathrm{TM}}$ as a clearing agent prior to coverslipping (Group C).

When UltraClear ${ }^{\mathrm{TM}}$ was used as both dewaxing and clearing agents prior to coverslipping, the results are comparable with xylene as the adequacy for Group D was $80 \%$. This finding is in line with Groups B and C. However, sections of tissues processed using UltraClear ${ }^{\mathrm{TM}}$ as dewaxing and clearing agents prior to coverslipping showed significant findings when compared to xylene (Group E). It is important to note that the blocks in Group E were previously processed with UltraClear ${ }^{\mathrm{TM}}$ as mentioned in our previous study. ${ }^{[6]}$ Nuclear and cytoplasm staining, cell morphology, clarity, and uniformity of staining were not well preserved. This might indicate that UltraClear ${ }^{\mathrm{TM}}$ processing has affected the efficiency of UltraClear ${ }^{\mathrm{TM}}$ in deparaffinization and clearing prior to coverslipping.

Since no other studies have evaluated UltraClear ${ }^{\mathrm{TM}}$ in an independent laboratory setting, we report the findings of other substitutes. Furthermore, the publications regarding xylene alternatives to dewaxing and clearing 
Table 4: Evaluation of UltraClear ${ }^{\mathrm{TM}}$ as dewaxing and clearing agents prior to coverslipping

\begin{tabular}{|c|c|c|c|c|c|}
\hline & $\begin{array}{c}\text { Group } \\
\text { A, } n\end{array}$ & $\begin{array}{c}\text { Group } \\
\text { B, } n\end{array}$ & $\begin{array}{c}\text { Group } \\
\text { C, } n\end{array}$ & $\begin{array}{c}\text { Group } \\
\mathrm{D}, n\end{array}$ & $\begin{array}{c}\text { Group } \\
\mathrm{E}, n\end{array}$ \\
\hline \multicolumn{6}{|l|}{ Nuclear staining } \\
\hline Adequate & 86 & 77 & 74 & 70 & 69 \\
\hline Inadequate & 14 & 23 & 26 & 30 & 31 \\
\hline$P$ & & 0.101 & 0.034 & 0.497 & 0.004 \\
\hline \multicolumn{6}{|c|}{ Cytoplasm staining } \\
\hline Adequate & 95 & 87 & 89 & 96 & 85 \\
\hline Inadequate & 5 & 13 & 11 & 4 & 15 \\
\hline$P$ & & 0.048 & 0.118 & 0.733 & 0.018 \\
\hline \multicolumn{6}{|l|}{ Cell morphology } \\
\hline Preserved & 82 & 71 & 81 & 66 & 58 \\
\hline Unpreserved & 18 & 29 & 19 & 34 & 42 \\
\hline$P$ & & 0.067 & 0.856 & 0.010 & 0.000213 \\
\hline \multicolumn{6}{|c|}{ Clarity of staining } \\
\hline Present & 85 & 71 & 61 & 78 & 73 \\
\hline Not present & 15 & 29 & 39 & 22 & 27 \\
\hline$P$ & & 0.017 & 0.000132 & 0.202 & 0.037 \\
\hline \multicolumn{6}{|c|}{ Uniformity of staining } \\
\hline Present & 83 & 82 & 81 & 78 & 70 \\
\hline Not present & 17 & 18 & 19 & 22 & 30 \\
\hline$P$ & & 0.852 & 0.713 & 0.372 & 0.03 \\
\hline
\end{tabular}

Group $\mathrm{A}=$ Standard method, Group B=UltraClear ${ }^{\mathrm{TM}}$ as a dewaxing agent, Group $\mathrm{C}=\mathrm{U}$ ltraClear ${ }^{\mathrm{TM}}$ as a clearing agent prior to coverslipping, Group $\mathrm{D}=\mathrm{U}$ ltraClear ${ }^{\mathrm{TM}}$ as both dewaxing and clearing agents prior to coverslipping (xylene-processed blocks), Group E=Evaluates UltraClear ${ }^{\mathrm{TM}}$ as dewaxing and clearing agents prior to coverslipping (UltraClear ${ }^{\mathrm{TM}}$-processed blocks). Number of slides in each group is 100

Table 5: Overall adequacy for UltraClearTM

\begin{tabular}{lcccccc}
\hline & Group A & Group B & Group C & Group D & Group E \\
\hline Adequate & 89 & 79 & 83 & 80 & 76 \\
Inadequate & 11 & 21 & 17 & 20 & 24 \\
$P$ & & 0.054 & 0.221 & 0.079 & 0.016 \\
\hline
\end{tabular}

Group $\mathrm{A}=$ Standard method, Group $\mathrm{B}$ evaluates UltraClear ${ }^{\mathrm{TM}}$ as a dewaxing agent, Group $C$ evaluates UltraClear ${ }^{\mathrm{TM}}$ as a clearing agent prior to coverslipping, Group D evaluates UltraClear ${ }^{\mathrm{TM}}$ as both dewaxing and clearing agents prior to coverslipping (xylene-processed blocks). Group E=UltraClear'M as dewaxing and clearing agents prior to coverslipping (UltraClear ${ }^{\mathrm{TM}}$-processed blocks). Number of slides in each group is 100

prior to coverslipping agents in the literature are very limited. Nontoxic xylene substitute called SBO was suggested to be a safe efficient substitute of xylene and may replace xylene without losing valuable diagnostic information. In their 6-month clinical practice, SBO was equally effective or superior to xylene when it comes to clearing and dewaxing. ${ }^{[11]}$

Cedarwood oil at $8 \%$, being nontoxic, noninflammable, nonhazardous, and easy to handle, produced good quality staining with sufficient clarity and uniformity of staining in comparison with xylene. ${ }^{[12]}$

Bleached palm oil, being nontoxic, noninflammable, nonhazardous, biodegradable, economic, easy to handle, and readily available, showed a minor difference in terms of transparency and quality of histological staining when compared with xylene. ${ }^{[1]}$ Similar findings were reported

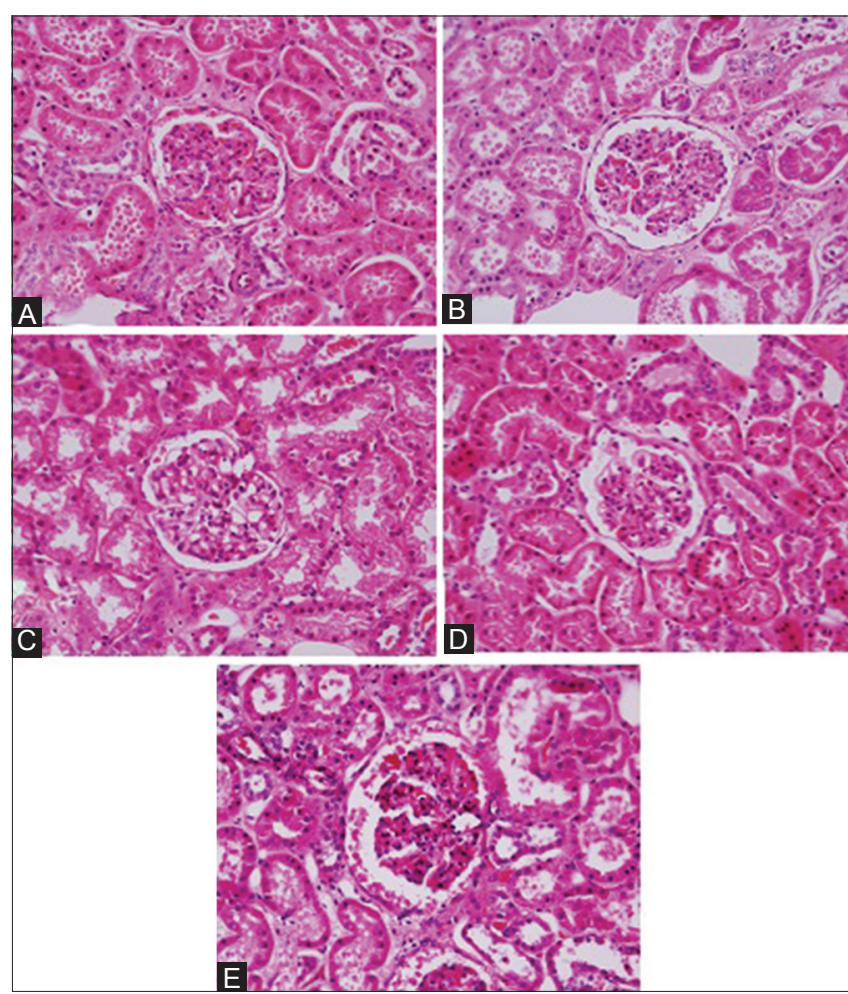

Figure 1: Nuclear staining in kidney tissues. Group $A$ is the standard method. Group B evaluates UltraClear ${ }^{\mathrm{TM}}$ as a dewaxing agent. Group C evaluates UltraClear ${ }^{\mathrm{TM}}$ as a clearing agent prior to coverslipping. Group $D$ evaluates UltraClear ${ }^{\mathrm{TM}}$ as both dewaxing and clearing agents prior to coverslipping (xylene-processed blocks). Group E evaluates UltraClear ${ }^{\mathrm{TM}}$ as dewaxing and clearing agents prior to coverslipping (UltraClear ${ }^{\mathrm{TM}}$-processed blocks) ( $\mathrm{H}$ and $\mathrm{E}, \mathrm{x} 40)$

with coconut and olive oils as substitutes for xylene during clearing and dewaxing. ${ }^{[13]}$

Dishwashing soap at $1.7 \%$, being nontoxic, cheap, easy to handle, and dispose, is an effective alternative dewaxing agent to xylene. ${ }^{[14]}$ Another study found that $1.7 \%$ dishwashing liquid soap at $90^{\circ} \mathrm{C}$ showed similar overall quality of tissue sections in deparaffinization when compared with xylene. ${ }^{[15]}$ Similar findings were also reported. ${ }^{[3,4,9]}$ In addition, dishwashing solution at $1.5 \%$ and $95 \%$ diluted lemon water can admittedly substitute xylene as deparaffinizing agents in $\mathrm{H}$ and E procedure. ${ }^{[16]}$

The approximate turnaround time taken for all groups is almost similar. The procedure takes about $46 \mathrm{~min}$. This finding is in line with other reported study, but they used a dishwashing soap as a dewaxing agent. ${ }^{[15]}$ However, the cost of UltraClear ${ }^{\mathrm{TM}}$ is double the xylene. There is only one sole agent that sells UltraClear ${ }^{\mathrm{TM}}$ in Oman. In addition, the use of UltraClear ${ }^{\mathrm{TM}}$ is not yet common. More important is the safety of workers in histopathology laboratories.

Few limitations of our study are worth noting. First, detailed information of different types of cells and tissues are absent. Second, cytologic staining such as Pap stain 
is not tested. Finally, UltraClear ${ }^{\mathrm{TM}}$ was not evaluated on immunohistochemical and cryostat section staining slides.

\section{Conclusion}

UltraClear ${ }^{\mathrm{TM}}$ is less toxic, less flammable, more friendly to the environment, and easier to handle than xylene but expensive. The findings of this study show that UltraClear ${ }^{\mathrm{TM}}$ is a promising dewaxing agent. It is also a good clearing agent for use prior to coverslipping in histopathology laboratory. Cost-benefit balance between safety of laboratory workers in histopathology, good quality staining, and cost-effective strategy needs to be further studied.

\section{Acknowledgments}

We would like to thank all staff in the Pathology Department at Sultan Qaboos University Hospital, Muscat, Sultanate of Oman, for their cooperation and help in providing the specimens.

\section{Financial support and sponsorship \\ Nil.}

\section{Conflicts of interest}

There are no conflicts of interest.

\section{References}

1. Udonkang M, Eluwa M, Ekanem A, Sharma TB, Asuquo OR, Akpantah AO. Bleached palm oil as substitute for xylene in histology. J Pharm Clin Res 2014;8:8-17.

2. Material Safety Data Sheet (MSDS) for Xylene. Fisher Scientific. Available from: https://www.fscimage.fishersci.com/ msds/25150.htm. [Last accessed on 2018 Jul 20].

3. Buesa RJ. Mineral oil: The best xylene substitute for tissue processing yet? J Histotechnol 2000;23:143-9.
4. Ankle MR, Joshi PS. A study to evaluate the efficacy of xylene-free hematoxylin and eosin staining procedure as compared to the conventional hematoxylin and eosin staining: An experimental study. J Oral Maxillofac Pathol 2011;15:161-7.

5. Material Safety Data Sheet (MSDS) for UltraClear ${ }^{\mathrm{TM}}$. Avantor Performance Materials B.V. Available from: http://www. avantormaterials.com / documents / MSDS / DEV / GB / MSDS_3905_GB.pdf. 2010. [Last accessed on 2018 Jul 15].

6. Alwahaibi N, Aljaradi S, Alazri H. Alternative to xylene as a clearing agent in histopathology. J Lab Physicians 2018;10:189-93.

7. Bancroft JD, Gamble M. Theory and Practice of Histological Techniques. $6^{\text {th }}$ ed. Philadelphia, USA: Churchill Livingstone Elsevier; 2008. p. 126-7.

8. Singh A, Hunasgi S, Koneru A, Vanishree M, Ramalu S, Manvikar V, et al. Comparison of honey with ethanol as an oral cytological fixative: A pilot study. J Cytol 2015;32:113-7.

9. Pandey P, Dixit A, Tanwar A, Sharma A, Mittal S. A comparative study to evaluate liquid dish washing soap as an alternative to xylene and alcohol in deparaffinization and hematoxylin and eosin staining. J Lab Physicians 2014;6:84-90.

10. Buesa RJ, Peshkov MV. Histology without xylene. Ann Diagn Pathol 2009;13:246-56.

11. Kunhua W, Chuming F, Tao L, Yanmei Y, Xin Y, Xiaoming Z, et al. A novel non-toxic xylene substitute (SBO) for histology. Afr J Tradit Complement Altern Med 2012;9:43-9.

12. Indu S, Ramesh V, Indu PC, Prashad KV, Premalatha B, Ramadoss $\mathrm{K}$, et al. Comparative efficacy of cedarwood oil and xylene in hematoxylin and eosin staining procedures: An experimental study. J Nat Sci Biol Med 2014;5:284-7.

13. Bruun Rasmussen B, Norring Hjort K, Mellerup I, Sether G, Christensen N. Vegetable oils instead of xylene in tissue processing. APMIS 1992;100:827-31.

14. Negi A, Puri A, Gupta R, Chauhan I, Nangia R, Sachdeva A, et al. Biosafe alternative to xylene: A comparative study. J Oral Maxillofac Pathol 2013;17:363-6.

15. Metgud R, Astekar MS, Soni A, Naik S, Vanishree M. Conventional xylene and xylene-free methods for routine histopathological preparation of tissue sections. Biotech Histochem 2013;88:235-41.

16. Ananthaneni A, Namala S, Guduru VS, Ramprasad VV, Ramisetty SD, Udayashankar U, et al. Efficacy of 1.5\% dish washing solution and $95 \%$ lemon water in substituting perilous xylene as a deparaffinizing agent for routine $\mathrm{H}$ and $\mathrm{E}$ staining procedure: A short study. Scientifica (Cairo) 2014;2014:707310. 